\title{
Cloning and characterization of the ecto-nucleotidase NTPDase 3 from rat brain: Predicted secondary structure and relation to other members of the E-NTPDase family and actin
}

\author{
Thomas Vorhoff ${ }^{1}$, Herbert Zimmermann ${ }^{1}$, Julie Pelletier ${ }^{2}$, Jean Sévigny ${ }^{2} \&$ Norbert Braun $^{1}$ \\ ${ }^{1}$ Biocenter, AK Neurochemistry, J.W. Goethe-University, Frankfurt am Main, Germany; ${ }^{2}$ Centre de Recherche \\ en Rhumatologie et Immunologie, Sainte-Foy, Québec, Canada
}

Received 5 November 2004; accepted in revised form 12 January 2005

Key words: actin, apyrase, ATP, ectonucleotidase, NTPDase, PC12 cell

\begin{abstract}
The protein family of ecto-nucleoside triphosphate diphosphohydrolases (E-NTPDase family) contains multiple members that hydrolyze nucleoside $5^{\prime}$-triphosphates and nucleoside $5^{\prime}$-diphosphates with varying preference for the individual type of nucleotide. We report the cloning and functional expression of rat NTPDase3. The rat brain-derived cDNA has an open reading frame of $1590 \mathrm{bp}$ encoding 529 amino acid residues, a calculated molecular mass of $59.1 \mathrm{kDa}$ and predicted N- and C-terminal hydrophobic sequences. It shares $94.3 \%$ and $81.7 \%$ amino acid identity with the mouse and human NTPDase3, respectively, and is more closely related to cell surface-located than to the intracellularly located members of the enzyme family. The NTPDase 3 gene is allocated to chromosome 8q32 and organized into 11 exons. Rat NTPDase 3 expressed in CHO cells hydrolyzed both nucleoside triphosphates and nucleoside diphosphates with hydrolysis ratios of ATP:ADP of 5:1 and UTP:UDP of 8:1. After addition of ATP, ADP is formed as an intermediate product that is further hydrolyzed to AMP. The enzyme is preferentially activated by $\mathrm{Ca}^{2+}$ over $\mathrm{Mg}^{2+}$ and reveals an alkaline $\mathrm{pH}$ optimum. Immunocytochemistry confirmed expression of heterologously expressed NTPDase 3 to the surface of CHO cells. PC12 cells express endogenous surface-located NTPDase3. An immunoblot analysis detects NTPDase3 in all rat brain regions investigated. An alignment of the secondary structure domains of actin conserved within the actin/HSP70/sugar kinase superfamily to those of all members of the NTPDase family reveals apparent similarity. It infers that NTPDases share the two-domain structure with members of this enzyme superfamily.
\end{abstract}

Abbreviations: ACR - apyrase conserved region; $\mathrm{CHO}$ - Chinese hamster ovary; ConA - concanavalin A; Cy3 - indocarbocyanin-3; DAPI - 2-(4-amidinophenyl)-6-indolecarbamidine dihydrochloride; DMEM - Dulbecco's modified Eagle's medium; EDTA - ethylenediaminetetraacetic acid; E-NTPDase - ecto-nucleoside triphosphate diphosphohydrolase; EST expressed sequence tag; NGF - nerve growth factor; PC12-pheochromocytoma 12; PCR-polymerase chain reaction; $\mathrm{ORF}$ - open reading frame

\section{Introduction}

The protein family of ecto-nucleoside triphosphate diphosphohydrolases (E-NTPDase family) contains multiple members that differ regarding tissue distribution, cellular location and substrate specificity. They hydrolyze nucleoside $5^{\prime}$-triphosphates and nucleoside $5^{\prime}$-diphosphates with varying preference for the individual type of nucleotide. Depending on subtype their catalytic site may be in ectoposition facing the extracellular medium or it may be

Correspondence to: Dr Norbert Braun, AK Neurochemie, Zoologisches Institut, Biozentrum der J.W. Goethe-Universität, Marie-Curie-Str. 9, 60439 Frankfurt am Main, Germany. Tel: +49-69-798-29604; Fax: +4969-798-29606; E-mail: n.braun@zoology.uni-frankurt.de located in the lumen of intracellular organelles. Whereas the surface-located members of the protein family are thought to be involved mainly in the control of ligand availability for $\mathrm{P} 2$ receptors, the functional role of the intracellular members is less defined $[1,2]$.

The gene family has members within vertebrates, invertebrates, plants, yeast and protozoans but has not been identified in bacteria (references in [1, 3-5]). Hallmarks of all E-NTPDases are five highly conserved sequence domains ('apyrase conserved regions,' $[3,4,6]$ ) that presumably are involved in the catalytic cycle. Interestingly, E-NTPDases share two common sequence motifs with members of the actin/HSP70/sugar kinase superfamily, the actin-HSP 70-hexokinase $\beta$ - and $\gamma$-phosphate binding motif $[(\mathrm{I} / \mathrm{L} / \mathrm{V}) \mathrm{X}(\mathrm{I} / \mathrm{L} / \mathrm{V} / \mathrm{C}) \mathrm{DXG}(\mathrm{T} / \mathrm{S} / \mathrm{G})(\mathrm{T} / \mathrm{S} / \mathrm{G}) \mathrm{XX}(\mathrm{R} / \mathrm{K} / \mathrm{C})][3$, 7-9], with the DXG sequence strictly conserved. 
In contrast to the members of the actin/HSP70/sugar kinase superfamily, mammalian E-NTPDases are membrane-anchored proteins with one or two transmembrane domains. NTPDase5 and NTPDase6 contain a single predicted N-terminal hydrophobic domain. They are located to the endoplasmic reticulum or Golgi apparatus respectively but they can also be released in soluble form from transfected cells [10-12]. They preferentially hydrolyze nucleoside diphosphates. The two forms of NTPDase4, that differ by alternative splicing, have predicted $\mathrm{N}$ - and Cterminal transmembrane domains and were allocated to the Golgi apparatus (UDPase) [13] and to lysosomal/autophagic vacuoles (LALP70) [14, 15], respectively. The Golgi enzyme hydrolyzes a number of nucleoside $5^{\prime}$-di and triphosphates but not ATP and ADP. NTPDase7 [16] is localized to intracellular organelles and hydrolyses a variety of nucleoside triphosphates with the exception of ATP. In mammals, four different surface-located subtypes of ENTPDases have been cloned and characterized. They share a membrane topography with $\mathrm{N}$ - and C-terminal transmembrane domains: NTPDase1 [17-20], NTPDase2 [8, 21], NTPDase3 [9, 22, 23], and most recently NTPDase8 [24]. They all hydrolyze nucleoside tri- and diphosphates but differ significantly in catalytic properties [25].

Surface-located mammalian E-NTPDases have been cloned and characterized mainly from rat, human and mouse tissues. Since these enzymes vary regarding substrate preference and product pattern formation it is necessary to compare the catalytic properties of individual enzymes within the same species. We have previously cloned and characterized rat NTPDase 1 and rat NTPDase2 $[8,20]$. Here we report the cloning and characterization of rat NTPDase3. We analyze its functional properties and tissue distribution in brain and compare some of the key properties of primary and secondary structure to those of other members of the gene family.

\section{Materials and methods}

\section{Materials}

Rat brain cDNA library SuperScript ${ }^{\mathrm{TM}}$, reverse transcriptase SuperScript ${ }^{\mathrm{TM}}$ II, Trizol ${ }^{\mathrm{TM}}$ reagent, oligo(dT)-cellulose, fetal calf serum, horse serum, DMEM (Dulbecco's modified Eagle's medium), penicillin and streptomycin, and trypsin/EDTA solution was obtained from Invitrogen (Karlsruhe, Germany). The cell culture medium Ham's F12 was from PAA Laboratories (Cölbe, Germany). Sawady Pwo DNA Polymerase was from PeqLab (Erlangen, Germany). Nucleoside triphosphate and diphosphate sodium salts, 2-(4-amidinophenyl)-6-indolecarbamidine dihydrochloride (DAPI), $\beta$-subunit of nerve growth factor (NGF) and proteinase $\mathrm{K}$ were purchased from SigmaAldrich (Taufkirchen, Germany). Concanavalin A (ConA)-sepharose, desoxycytidine $5^{\prime}\left[\alpha^{32} \mathrm{P}\right]$-triphosphate and the enhanced chemiluminescence system were from Amersham Biosciences (Freiburg, Germany). Protease inhibitors antipain, aprotinin, benzamidine, chymostatin, leupeptin, and pepstatin were obtained form Calbiochem (Schwalbach, Germany). Restriction endonucleases were purchased from MBI Fermentas (St. Leon-Rot, Germany) or Invitrogen (Karlsruhe, Germany). BOA protein marker was from Biomol (Hamburg, Germany) and protran nitrocellulose membranes from Schleicher \& Schuell (Dassel, Germany). Nucleobond AX 500 plasmid purification kit and NucleoTrap DNA purification kit were from Machery-Nagel (Düren, Germany). Synthetic oligonucleotides were obtained from BioSpring (Frankfurt, Germany), indocarbocyanin-3 (Cy3)-labeled anti-rabbit IgG antibody was from Dianova (Hamburg, Germany).

\section{cDNA library screening}

Total RNA from rat brain was isolated with Trizol reagent. For isolation of polyadenylated RNA from total RNA, oligo(dT)-cellulose was used according to the manufacturer's instructions. A cDNA library was synthesized with SuperScript ${ }^{\circledR}$ II from $0.5 \mu \mathrm{g}$ of mRNA with an oligo(dT) $)_{18}$ primer in accordance with the manufacturer's instructions. Two mouse sequences from expressed sequence tag (EST) databases (GenBank accession numbers bf302156 and w46136) were used for primer design. As a probe, a 288bp PCR fragment was amplified using forward primer 5'-CCGTCCCTGCTCCCAAGATTT-3', reverse primer 5'CAGGCACAGCAAGGCGATAGC- $3^{\prime}$, and the rat brain cDNA library as a template. For library screening, electrocompetent Escherichia coli DH5 $\alpha$ were transformed with a rat brain pCMV-SPORT 2 cDNA library amplified and plated on Luria-Bertani/ampicillin agar plates. The resulting transformants were screened by colony hybridization with the 288-bp cDNA fragment labeled with $\left[\alpha-{ }^{32} \mathrm{P}\right] \mathrm{dCTP}$ by PCR. Positive signal areas were amplified and rescreened for single positive colonies.

\section{cDNA sequencing and computational sequence analysis}

DNA sequencing was performed by Scientific Research and Development GmbH (Oberursel, Germany). Primer walking in both directions was employed for obtaining the complete full length sequence of the cDNA clone 3.1.1.1. The Omiga 2.0 sequence analysis program (Oxford Molecular Ltd., Oxford, UK) was used for assembling sequence fragments, translating DNA into amino acid sequences, generating hydrophobicity blots and amino acid alignment (CLUSTAL W algorithm). To align the amino acid sequences for the dendrogram, ClustalX 1.81 and for the graphic depiction BoxShade v3.31c were used. For prediction of transmembrane domains, the software TMHMM 2.0 (www.cbs.dtu.dk/services/TMHMM-2.0) was employed. For signal peptide and sorting analysis, SignalP 3.0 (www.cbs.dtu.dk/services/SingalP/) and PSORT II (http:// psort.nibb.ac.jp/form2.html) were used. The DNA and deduced amino acid sequences were analyzed for similarity to known sequences with the NCBI Blast Network service (www.ncbi.nlm.nih.gov/BLAST/). Protein motif search was 
performed using the prosite database (www.expasy.org/ prosite/). Secondary structure prediction of the amino acid sequences was performed with the SSpro tool (www.igb. uci.edu/tools/scratch/). The genomic library was screened using BLAST and the splice analysis of the genomic sequence was performed using the splice site analysis tool www.fruitfly.org/seq-tools/splice.html.

\section{Expression of recombinant proteins}

For recombinant expression, the EcoRI/NotI cDNA fragment of clone 3.1.1.1 was cloned into the EcoRI/NotI sites of pcDNA3. Chinese hamster ovary $(\mathrm{CHO})$ cells were cultured as previously described [8]. Cells were transfected by electroporation with the rat NTPDase3 pcDNA3 plasmid or with plasmids expressing rat NTPDase1 [20] or NTPDase2 [8] in electroporation buffer (in mM: 137 $\mathrm{NaCl}, 5 \mathrm{KCl}, 0.7 \mathrm{Na}_{2} \mathrm{HPO}_{4}, 6$ dextrose, 20 Hepes, $\mathrm{pH} 7.0$ ) using a BTX Electrocell manipulator 600. In control experiments cells were transfected with empty vector alone. Twenty-four hours after electroporation the culture medium of transfected $\mathrm{CHO}$ cells was exchanged to remove dead cells and debris.

\section{Preparation of membrane fractions}

Forty-eight hours after electroporation, the culture medium was removed, cells were washed twice with isotonic buffer (in mM: $140 \mathrm{NaCl}, 5 \mathrm{KCl}, 0.5$ EDTA, $20 \mathrm{MOPS}, \mathrm{pH} 7.4$ ) and scraped from the plates in ice cold homogenization buffer (in mM: 250 sucrose, 2 EDTA, 2 iodoacetamide, 30 MOPS, $\mathrm{pH}$ 7.4) containing a mixture of protease inhibitors (in $\mu \mathrm{g} / \mathrm{ml}$ : 2 chymostatin, 2 aprotinin, 1 pepstatin, 150 benzamidine, 2 antipain, 2 leupeptin). After centrifugation at $300 g_{\text {av }}$ cells were resuspended in homogenization buffer, homogenized, and centrifuged for $10 \mathrm{~min}$ at $300 \mathrm{~g}_{\mathrm{av}}$ at $4{ }^{\circ} \mathrm{C}$. The resulting supernatant was sonicated and subsequently centrifuged at $100,000 \mathrm{~g}_{\mathrm{av}}$ for $60 \mathrm{~min}$ at $4{ }^{\circ} \mathrm{C}$, pellets were resuspended in storage buffer (in $\mathrm{mM}: 2$ iodoacetamide, 25 Hepes, $\mathrm{pH} 7,4$ ) containing the protease inhibitor mixture and $50 \%(\mathrm{v} / \mathrm{v})$ glycerol, and stored at $-20{ }^{\circ} \mathrm{C}$ until further processing. For preparation of membrane fractions from various brain tissues, Wistar rats obtained from Charles River Wiga (Sulzfeld, Germany) were used. Animals were anaesthetized with $\mathrm{CO}_{2}$, decapitated, the brain was dissected, homogenized in five volumes of homogenization buffer containing the protease inhibitor mixture and further processed as described above.

\section{Measurement of nucleotidase activities}

Nucleotidase activity was determined by measuring the formation of Pi liberated from nucleotides [26]. Membrane fractions were incubated in phosphate-free solution containing $500 \mu \mathrm{M} \mathrm{CaCl}_{2}$ and $25 \mathrm{mM}$ Hepes (pH 7.4), and 500 $\mu \mathrm{M}$ nucleoside tri- or diphosphates, respectively, at $37^{\circ} \mathrm{C}$. Samples were heat-inactivated for $2 \mathrm{~min}$ at $95{ }^{\circ} \mathrm{C}$ prior to determination of inorganic phosphate. To investigate the dependence of enzyme activity on metal ions, 50-400 $\mu \mathrm{M}$ $\mathrm{CaCl}_{2}$ or $\mathrm{MgCl}_{2}$ was added, or $\mathrm{CaCl}_{2}$ and $\mathrm{MgCl}_{2}$ were replaced by $1 \mathrm{mM}$ EDTA. pH-dependency was determined using a combined buffer (25 mM Hepes and $50 \mathrm{mM}$ glycine) ranging from $\mathrm{pH} 3$ to 10 , containing $500 \mu \mathrm{M}$ ATP and $500 \mu \mathrm{M} \mathrm{CaCl}_{2}$ or $500 \mu \mathrm{M} \mathrm{MgCl}_{2}$. Catalytic activity of membrane fractions derived from cells transfected with the empty plasmid was subtracted from that obtained with cDNA-transfected cells. It was verified for each experimental condition applied that hydrolysis rates were constant $(10-30 \mathrm{~min})$. At the end of the reaction with nucleoside triphosphate it was ensured that less than $10 \%$ of the initial substrate was hydrolysed.

For determination of product formation following ATP hydrolysis $(250 \mu \mathrm{M}$ ATP, $250 \mu \mathrm{M} \mathrm{CaCl} 2)$, aliquots were collected at various time points and subjected to HPLC analysis. Following heat inactivation, samples were centrifuged at 20,000 $g_{\mathrm{av}}$ for $15 \mathrm{~min}\left(4^{\circ} \mathrm{C}\right)$. ATP, ADP, AMP, and adenosine were separated by a Sepsil $\mathrm{C}_{18}$ reverse phase column (Jasco, Groß-Umstadt, Germany) and eluted with the mobile phase, consisting of $50 \mathrm{mM}$ potassium-phosphate buffer ( $\mathrm{pH}$ 6.5), 6\% methanol and $5 \mathrm{mM}$ tetrabutylammonium hydrogen sulfate [20]. The absorbance at 260 $\mathrm{nm}$ was continuously monitored and nucleotide concentration was determined from the area under each absorbance peak.

\section{Immunoblotting}

The anti-rat NTPDase3 antibody (N3-3i4) was raised in rabbits by direct injection of the encoding NTPDase3pcDNA3 plasmid [27]. Previous to Western blot analysis of membrane fractions from transfected $\mathrm{CHO}$ cells or mouse brain tissues, NTPDase 3 was enriched using ConASepharose. The $100,000 g_{\text {av }}$ pellet was solubilized in ConAbuffer (in mM: $150 \mathrm{NaCl}, 2 \mathrm{MgCl}_{2}, 2 \mathrm{CaCl}_{2}, 2 \mathrm{MnCl}_{2}, 20$ Tris/ $\mathrm{HCl}, \mathrm{pH} 7.4$ ) containing $0.1 \%$ Triton $\mathrm{X}-100$. After an overnight incubation with ConA-Sepharose at $4{ }^{\circ} \mathrm{C}$, the ConA-Sepharose was washed several times with ConAbuffer containing $0.1 \%$ Triton $\mathrm{X}-100$, and protein was eluted with the same buffer containing $200 \mathrm{mM}$ methyl- $\alpha-$ D-mannopyranoside. For immunoblotting the ConA eluate was precipitated with $10 \%$ trichloroacetic acid and the pellet was resuspended in sample buffer without reducing agents. Polyacrylamide gel electrophoresis was carried out on minigels (10\% acrylamide). Immunoblotting using the polyclonal NTPDase3 antibody (dilution 1:4000) was performed using the Amersham enhanced chemiluminescence system according to the manufacturer's instructions.

\section{Immunofluorescence}

Transfected CHO cells (15,000 cells per well) or nontransfected PC12 cells (15,000 cells per well) were seeded onto poly-D-lysine-coated $(10 \mu \mathrm{g} / \mathrm{ml})$ glass cover slips (10 $\mathrm{mm}$ diameter) and cultured for 2 days ( $\mathrm{CHO}$ cells) or up to 14 days (PC12 cells) [28]. An aliquot of $50 \mathrm{ng} / \mathrm{ml}$ of NGF was added to PC12 cells every 3-4 days. For surface 
AGCGAGCGCAGCGCGCAGGTAAGGTCTGGACCGGCGCGCAGGAGTGGGATGGAGCAGCTGCCGGAGGCTTGAAGCACGGAGAGAAGGACCCGGCACAGC TTCCCACAGAGGACCGCAGGCTGGAGCCGAAACCCGGGGAGCTGGGCACTGTCATCTGACAGGCACAGGACACCGCGTCACGAAACGCCCCAAGACCTG

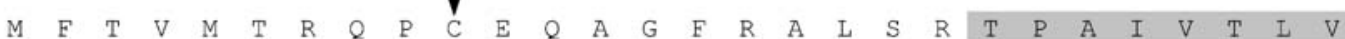
ATGTTACGGTGATGACCGCCAGCCCTGTGAACAGGCAGGCTTCAGGGCCCTCTCGAGGACTCCAGCCATCGTCACCTTGGTG

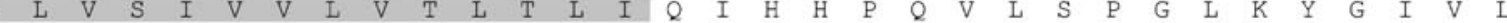
GTCCTGCTTGTGAGCATCGTGGTACTTGTGACACTTACACTCATCCAGATCCACCACCCACAGGTTCTCTCTCCAGGGCTGAAGTATGGAATCGTGTTG ACR 1

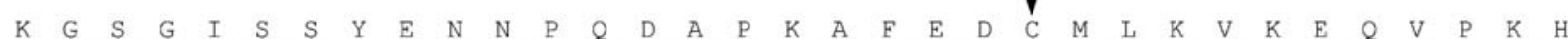
AAAGGCTCGGGAATCTCCAGCTATGAGAATAACCCCCAAGATGCCCCCAAAGCCTTTGAGGACTGCATGCTAAAGGTCAAGGAACAGGTCCCAAAGCAT

\section{ACR 2 \\ ACR 2}

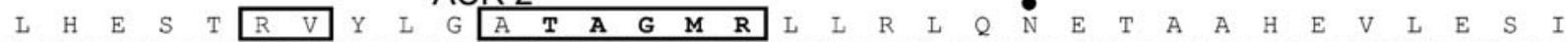
CTCCACGAATCCACCCGAGTTTACCTGGGGGCCACAGCTGGGATGCGCTTGCTGAGGTTGCAAAATGAGACAGCAGCTCATGAAGTCCTTGAGAGCATC ACR 3

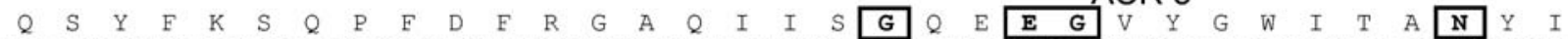
CAAAGCTACTTCAAGTCCCAGCCTTTTGATTTTAGGGGTGCTCAAATCATTTCTGGGCAAGAGGAAGGGGTGTATGGATGGATTACAGCCAACTATATA \begin{tabular}{llllllllllllllllllllllllll}
$M$ & $G$ & $N$ & $F$ & $L$ & $E$ & $K$ & $N$ & $L$ & $W$ & $H$ & $M$ & $W$ & $V$ & $H$ & $P$ & $H$ & $G$ & $V$ & $D$ & $T$ & $T$ & $G$ & $A$ & $I$ & $D$ \\
\hline
\end{tabular} ATGGGAAATTCCTGGAGAAGAACCTGTGGCACATGTGGGTGCACCCGCACGGAGTAGACACCACAGGAGCCCTGGATTTGGGCGGCGCCTCCACCCAG

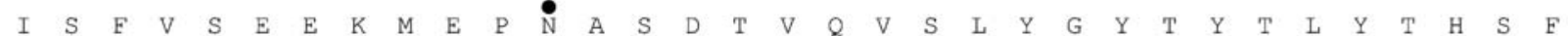
ATATCCTTTGTGTCGGAAGAGAAGATGGAGCCGAACGCCAGCGACACAGTGCAGGTGTCCTTGTACGGCTACACGTACACTCTCTACACGCACAGTTTC $\underset{C}{\nabla}$ CAGTGCTACGGCCGGAACGAAGCAGAGAAGAAGTTCCTGGCGATGCTTCTGCAGAGCCCTTCTACAGATGCCAACATCAGCAACCCCTGCTACCCTCAC

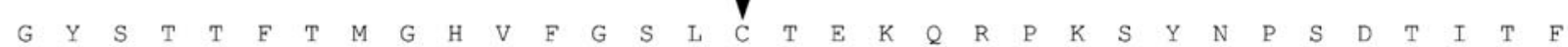
GGTTACAGCACCACCTTCACAATGGGCCACGTGTTTGGCAGCCTGTGCACGGAGAAGCAGAGGCCGAAGAGCTACAACCCCAGTGATACCATCACCTTC $\begin{array}{lllllllllllllllllllllllllllllllllll}T & G & T & G & D & P & Q & L & C & R & E & K & V & A & S & V & F & D & F & S & A & C & Q & E & Q & D & A & C & S & F & D & G & I\end{array}$ ACAGGAACTGGTGACCCACAGCTGTGCAGGGAGAAGGTGGCTTCTGTGTTTGACTTCAGTGCTTGCCAAGAGCAAGATGCCTGCTCCTTTGATGGCATT

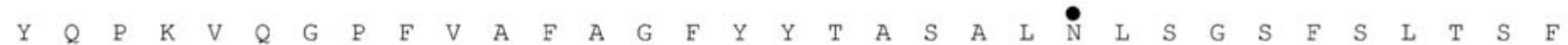
TACCAGCCCAAGGTTCAAGGGCCATTTGTGGCATTTGCAGGATTCTATTACACAGCCAGTGCCCTAAACCTCTCTGGAAGCTTCTCCCTTACCTCCTTC $\begin{array}{lllllllllllllllllllllllllllllllllll}\bullet & D & S & S & W & D & F & C & R & H & T & W & S & E & L & P & S & L & L & P & R & F & D & E & T & Y & A & R & S & Y & C & F & S\end{array}$ AATGACAGCAGCTGGGACTTCTGCAGACACACTTGGAGCGAGCTCCCGTCCCTGCTCCCCAGATTTGATGAGACGTATGCCCGGTCCTACTGCTTCTCA

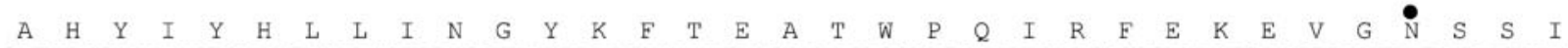
GCTCACTACATCTACCACTTGCTCATAAATGGATACAAATTCACTGAGGCAACTTGGCCTCAGATACGGTTTGAAAAAGAAGTGGGGAATAGCAGCATC ACR 5

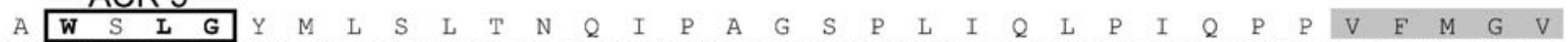
GCCTGGTCCCTAGGCTACATGCTCAGCTTGACCAACCAGATCCCAGCTGGAAGTCCGCTGATCCAACTACCCATACAGCCGCCGGTGTTTATGGGAGTC

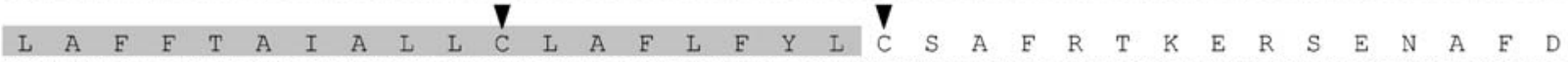
CTGGCCTTCTTCACGGCTATCGCCTTGCTGTGCCTGGCATTTCTTTTCTATCTATGTTCAGCATTTAGGACAAAGGAGCGCTCTGAGAATGCCTTTGAC Q A $A$ D D S D -

CAAGCAGTGGATTCTGATTGAATCTTCCA.AGCAGCACCTGGGGCCTGAGGCTGCCATAACCCACCAGAGTGACACCAGGCAGGGAGTGAAGTGGCTGCC

ACCAGGACCTGTCCCGTGGTTAAATGCCAAGATCACGTGCCTCTCAGCCACTGGTTCCTGCTGGAGCACCTCCTGGGAGTCCCTCAGCCACCCAGTGGG TGTTGCCTCTGCACCCTTCGTACCCGCTTGCTGATCCATTGGAAATCAAGGAGACACTGGCAGTTGGTGTCAAGGGCTTCACCCTGTCTCCTCCAGAGG ATGTTGAGAGTATAATTTAATTCGGAAGGATTGACCCAGGCTGGGTTTCCATTTTCTCTCCCTCCGTATTCCTCCTAGCAAGCTGCCCAGGAAGGCTCT GACTCAGACACTTCACCCTGTAGGTTTGACCTGTAGTTCCTTTGCAAGGCCTTCCAGTCTTTTAGTTGAGTGGGGCCCTATCTCAAGCCACTGATACTT TCTCTTAGCAATCTGGTAAGCACTAAGCTCCCTGACTGGTAGCTTGTAGCACTTAGCAGGGAAGATGATACCTTTTGAGACAGAGTTTCATGTAGCCCA TTCACTATGTAGCCAAGACCACTCACTAAGTAGCTCTTTTCCTGAGGGCTGCGATTATGGGTTGTGAGGTCCTGGGGGTGGAACCCCAGGGCTTCATGT ATGCTAGGCAATTATTCGACCAGCCTGAGTCACATGCCCAGCCTAAGAAGACTCACAGCCCCAAGGACAGACGGCCTCTGTCATGTAGGCAGAACCTGA AGCACAAAGCCACACAGGAGTCCCTAAGCTATTAGCTATTCTTATTTTGGAATGCCCACTTGGAGCTCTGGTCATGACTTTGCTACTTGGAGTATGTCA TCCTTGTGTAACTGCCTCCCCGCTGTTCCCTCTTGTAAATCGCACCCAGTGGACTGCACCAAGGTGGGAGGTTGGGGGTGGATCCCATTGACCTGACAA AGCTCCTGCCCAAGGTGCCTTTGGGCTATAACCATGTCGGCGTGTGCTTTCTTCCCACTGCTCCCACAGGTATCCAGGATCCTGTCCAAAAAACATCCC TACGTTTCTTCCTGCATAACTGCTTCCCAGCTTGACTTTGGGGAAAGAAAAGGCAGTCTTGGGAATGTTTGCTTCCAAAGTGGGATGGGAAAGGTCTTC AAACCCAGTAGCTACAAGCGACAAATCAATGCTGTGCCTCAAGGCTGGTCGTTGGCTCCATATGGAATAAACTATTATTTTTATTTTGCAATATGAAAA AAAAAAAAAAAAAAAAGG

Figure 1. DNA sequence and predicted protein sequence of rat NTPDase3. The five 'apyrase conserved regions' (ACR) are indicated by boxes and numbered. Cysteine residues and potential $\mathrm{N}$-glycosylation sites are indicated by arrow heads and filled circles, respectively. Predicted N- and Cterminal hydrophobic sequences are shaded. Amino acid residues conserved from NTPDase1 and NTPDase8 are in bold. In ACR 1 and ACR 4 these include the DXG-containing phosphate-binding motif and a conserved glycine in ACR 5. (GenBank accession number AJ437217.) 
labeling, the anti-rat NTPDase 3 antibody (1:1000) was applied to viable cells for $15 \mathrm{~min}$ at $37^{\circ} \mathrm{C}$ followed by repeated washing with phosphate-buffered saline (PBS, in mM: $137 \mathrm{NaCl}, 3 \mathrm{KCl}, 15 \mathrm{Na}^{+} / \mathrm{K}^{+}$phosphate buffer, $\mathrm{pH}$ 7.4) at room temperature, methanol fixation at $-20{ }^{\circ} \mathrm{C}$ and application of $\mathrm{Cy} 3$-conjugated anti-rabbit $\mathrm{IgG}$ antibody together with 2-(4-amidinophenyl)-6-indolecarbamidine dihydrochloride (DAPI, $1 \mu \mathrm{g} / \mathrm{ml}$ ). After immunolabeling, cells were mounted and investigated with an epifluorescence microscope equipped with an MCID 4 imaging analysis system (Imaging Research, St. Catherines, Canada).

\section{Results}

Cloning and sequencing of rat NTPDase 3

Two mouse EST sequences (GenBank accession numbers bf302156 and w46136) homologous to human NTPDase3 (GenBank accession number AF034840) were used for primer design. With these primers a 288-bp fragment was amplified from rat brain cDNA by RT-PCR. Using colony hybridization, six positive cDNA clones were isolated from a rat brain pCMV-SPORT 2 cDNA library using the 288bp radiolabeled PCR fragment as a probe. One of the clones (3.1.1.1) was completely sequenced in both directions by primer walking. The cDNA of clone 3.1.1.1 had a length of $3087 \mathrm{bp}$ and revealed a deduced open reading frame (ORF) of $1590 \mathrm{bp}$ from nt 214 to 1803. The ORF encodes 529 amino acid residues with a calculated protein molecular mass of $59.1 \mathrm{kDa}$, and an isoelectric point of 5.75. A polyadenylation signal (AAUAAA) was located 24 nucleotides upstream of the poly(A) tail. The deduced amino acid sequence (Figure 1) contains seven potential $N$-glycosylation sites located to the extracellular domain. It reveals 13 cysteine residues of which 10 are located

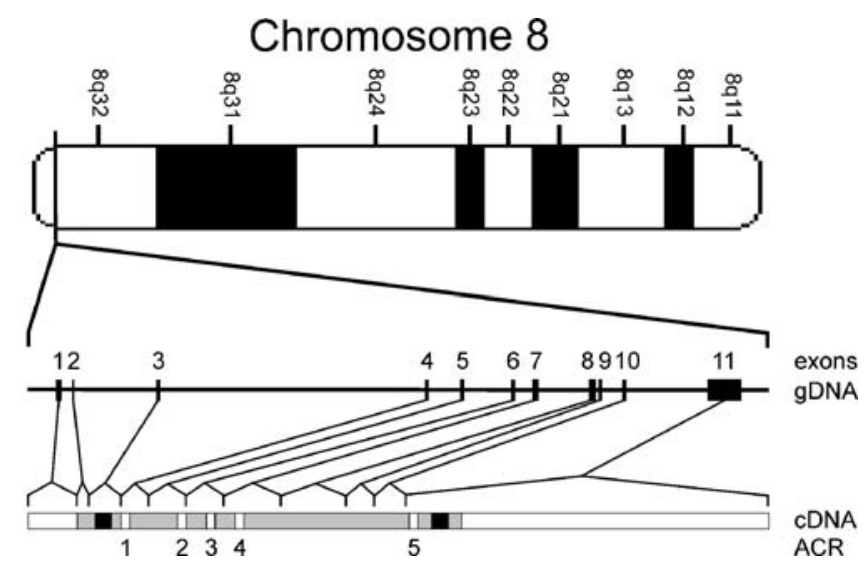

Figure 2. Chromosomal localization of rat NTPDase3 and intron-exon structure. The upper part of the figure depicts the position of the NTPDase3 gene in chromosome $8 \mathrm{q} 32$. The lower part enlarges the genomic sequence of NTPDase3. Upper line: Exons are numbered and indicated by black boxes in relation to the length of the encoded sequences (gDNA fragment $=31 \mathrm{~kb}$ ). The lower line indicates the length of individual exons. The ORF is shaded. The position of ACRs 1 to 5 is indicated by white boxes, that of the two putative transmembrane domains by black boxes.

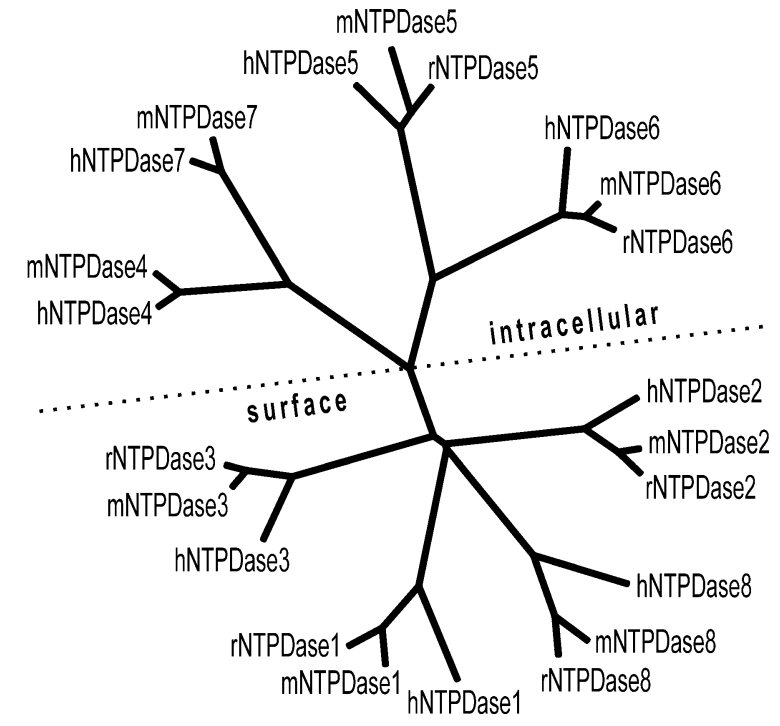

Figure 3. Hypothetical phylogenetic tree derived for 22 selected members of the E-NTPDase family (NTPDase1 to NTPDase8) from rat $(r)$, human $(h)$ and mouse $(m)$. Amino acid sequences were aligned using ClustalX 1.81 and the dendrogram was prepared using TreeView 1.6.6. The length of the lines indicates the differences between amino acid sequences. The graph depicts a clear separation between intracellular (top) and surface-located NTPDases (bottom). Protein sequences are derived from mRNA sequences. The GenBank accession numbers of the sequences are as follows: (NTPDase1, Homo sapiens (S73183), Mus musculus (AF037366), Rattus norvegicus (U81295); NTPDase2, Homo sapiens (U91510), Mus musculus (AF042811), Rattus norvegicus (Y11835); NTPDase3, Homo sapiens (AF034840), Mus musculus (AY376710), Rattus norvegicus (AJ437217); NTPDase4, Homo sapiens (AF016032), Mus musculus (AK004761); NTPDase5, Homo sapiens (AF039918), Mus musculus (AF006482), Rattus norvegicus (BC62044); NTPDase6, Homo sapiens (AF039916), Mus musculus (NM_172117), Rattus norvegicus (AJ277748); NTPDase7, Homo sapiens (AK055540), Mus musculus (AF288221); NTPDase8, Homo sapiens (AY430414), Mus musculus (AY364442), Rattus norvegicus (AY536920).

to the extracellular domain, five potential protein kinase $\mathrm{C}$ phosphorylation sites and six potential casein kinase II phosphorylation sites. The hydrophobicity analysis predicts two strong hydrophobic stretches in the polypeptide chain, close to the $\mathrm{N}$ - and C-terminal ends (Figure 1). These regions represent predicted transmembrane domains with the $\mathrm{N}$ - and $\mathrm{C}$-terminus at the cytosolic side, separated by a large extracellular loop. This corresponds to the membrane topography of the known plasma membrane-located NTPDases [1].

A homology search using the rat genomic sequence database localized the NTPDase3 gene (Entpd3) to chromosome 8q32 (Genebank Acc.-No. NW_047803 RN8_2327: 3551369-3651370). Alignment of the rat NTPDase 3 cDNA against the rat genomic sequence revealed that Entpd3 covers $31012 \mathrm{bp}$ and is organized into 11 exons and 10 introns of which exons 2 to 11 encode the open reading frame (Figure 2). Exon 1 contains most of the $5^{\prime}$-untranslated region of the NTPDase 3 cDNA. The ACRs 1-4 are contained in exons 4, 5, 6 and 7, respectively, whereas ACR5 is contained in exon 11. The N-terminal transmembrane domain is situated in exon 3 whereas the C-terminal transmembrane domain and the stop codon are localized in exon 11. 


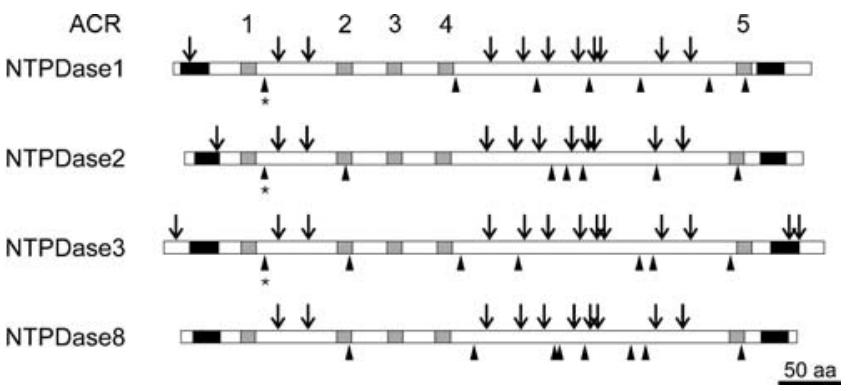

Figure 4. Alignment of potential $N$-glycosylation sites and of cysteine residues in surface-located NTPDases. All sequences from Rattus norvegicus. Potential $N$-glycosylation sites are indicated by arrow heads, cysteine residues by arrows, and the five 'apyrase conserved regions' (ACR) by shading. The asterisk marks the only potential glycosylation site conserved between NTPDase1, NTPDase2 and NTPDase3. Predicted $\mathrm{N}$ - and C-terminal hydrophobic sequences are indicated by black boxes. For GenBank accession numbers see legend of Figure 3.

\section{Relation to other members of the E-NTPDase family}

The deduced amino acid sequence of the cloned rat NTPDase 3 shares $94.3 \%$ and $81.7 \%$ amino acid identity, respectively, with the mouse [23] and human [22] orthologs. Compared to other rat members of the E-NTPDase family, NTPDase 3 is most closely related to cell surfacelocated NTPDase2, NTPDase8, and NTPDase1 (36.9\%,
$36.3 \%, 35.1 \%$ identity, respectively). It is more distantly related to the intracellularly located rat NTPDase 5 and rat NTPDase6 (17.8\% and 15.2\% identity, respectively). The graphic depiction of a multiple sequence alignment of 22 mammalian members of the E-NTPDase family illustrates the subdivision of the family into intracellular and surfacelocated enzymes (Figure 3). Whereas the origins of the branches for the four surface-located members (NTPDase1, NTPDase2, NTPDase3, NTPDase8) are located at close distance to each other, the intracellular members are divided into two sub-branches. One sub-branch consists of NTPDase 4 and NTPDase 7 that share their membrane topology with the surface-located enzymes. The other subbranch consisting of NTPDase5 and NTPDase6 is characterized by a single N-terminal hydrophobic domain, which can be cleaved resulting in the formation of a soluble enzyme [2].

The surface-located members of the E-NTPDase family share 10 cysteine residues in comparable location within the sequence (Figure 4). Of these, two residues are situated between ACR1 and ACR2 and eight residues between ACR4 and ACR5. The closely related NTPDase1 and NTPDase 2 possess an additional cysteine residue located at the N-terminal transmembrane domain. In contrast, rat NTPDase 3 has one additional cysteine residue at the Nterminal intracellular domain and two cysteine residues at

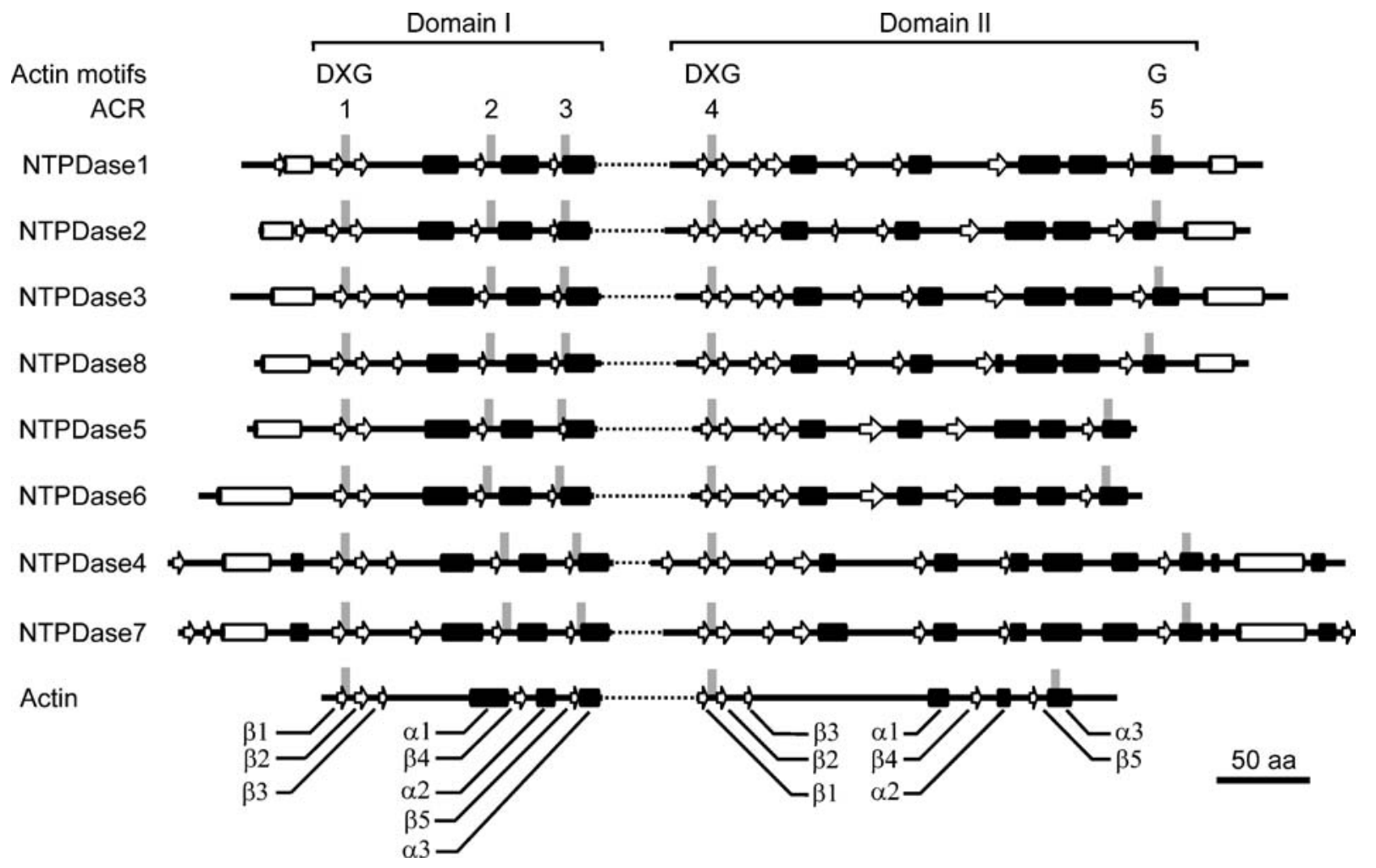

Figure 5. Comparison of the secondary structure conserved between members of the actin/HSP70/sugar kinase superfamily (as depicted for rabbit actin, Swiss-Prot accession P29751) with the predicted secondary structure of the E-NTPDase family members. Secondary structure prediction was performed with the SSpro tool. $\alpha$-Helices are indicated by black boxes, $\beta$-strands by arrows and putative transmembrane domains by white boxes. ACRs 1 to 5 are indicated by shading. The alignment of the first domain is oriented around the DXG motif in ACR 1 of NTPDases and the corresponding motif in actin. Correspondingly, the second domain is aligned to the DXG motif in ACR 4 and the corresponding motif in actin. In order to emphasize the repetition in topology, the sequence has been interrupted by dots. The conserved $\alpha$-helices and $\beta$-strands in actin are consecutively numbered for each of the two domains. All NTPDase sequences are from rat with the exception of NTPDase4 and NTPDase7 which are from mouse. For GenBank accession numbers see legend of Figure 3. 
the C-terminal transmembrane domain. The plasma membrane-located E-NTPDases possess seven to eight predicted N-linked glycosylation sites. Their distribution within the protein sequence is similar but not identical. Only the $N$-glycosylation site between ACR1 and ACR2 (N81 in rat NTPDase3) is conserved between NTPDase1, NTPDase2, and NTPDase3 (rat, mouse, and human sequences). This site is essential for full enzymatic activity of human NTPDase3 [29] but it does not exist in NTPDase8 (mouse, human). The majority of the potential $N$-glycosylation sites is situated between ACR4 and ACR5.

\section{Secondary structure prediction and relation to the actin/HSP70/sugar kinase superfamily}

The similarities in the distribution of the ACRs between the members of the E-NTPDase family imply structural conservation relevant for catalytic activity. It has previously been emphasized that NTPDases share two nucleotide binding motifs with the actin/HSP70/sugar kinase superfamily [3, 8, 9]. Members of this superfamily share little overall sequence identity except for the strictly conserved DXG motifs in the consensus sequence. These motifs can be identified in ACR1 and ACR4 of all ENTPDases.

There are, however, apparent similarities in the secondary structure of members of the actin/HSP70/sugar kinase superfamily. Its members possess two major domains with similar topology $\left(\beta_{1} \beta_{2} \beta_{3} \alpha_{1} \beta_{4} \alpha_{2} \beta_{5} \alpha_{3}\right)$ that fold into a pocket for substrate binding [30-32]. The two domains presumably are the result of gene duplication. Each of these domains is composed of two subdomains ( $a$ and $b$ ). The opposing subdomains Ia and IIa share the same basic topology $\left(\beta_{1} \beta_{2} \beta_{3} \alpha_{1} \beta_{4} \alpha_{2} \beta_{5} \alpha_{3}\right)$ that is involved in nucleotide binding. The nucleotide binding site bridges both domains. The two subdomains Ib and IIb (formed by sequences in between $\beta_{3}$ and $\alpha_{1}$ ) are different from one subfamily to another [30]. Figure 5 shows an alignment of the secondary structure conserved between members of the actin/HSP70/ sugar kinase superfamily, as depicted for actin, with the predicted secondary structure of NTPDase1 to NTPDase8. The atomic structure of actin has previously been revealed $[33,34]$. At the N-terminus, the alignment is oriented around the DXG motif in ACR1 of NTPDases and the corresponding DXG motif in actin. The two $\beta$ strands around ACR1 of E-NTPDases would thus correspond to $\beta_{1}$ and $\beta_{2}$ of actin. The overall pattern of conserved actin secondary structure $\left(\alpha_{1} \beta_{4} \alpha_{2} \beta_{5} \alpha_{3}\right)$ is very similar to that of NTPDases. This $\beta_{1} \beta_{2} \beta_{3} \alpha_{1} \beta_{4} \alpha_{2} \beta_{5} \alpha_{3}$ topology is repeated in the second domain of actin. Similar to actin, the conserved ACR4 of the NTPDases (second DXG motif) is embedded between two $\beta$ strands. Taking further into consideration the distance between $\beta_{3}$ and $\alpha_{1}$ in the second domain of actin and assuming that the glycine residue conserved between E-NTPDases in ACR5 (Gly462 in NTPDase3) corresponds to the conserved glycine residue in $\alpha_{3}$ of the actin/HSP70/sugar kinase superfamily (Gly342 in actin), the secondary structure of the C-terminal half of ENTPDases may also be aligned.

The degree of similarity in secondary structure mirrors the phylogenetic distance between sequences (comp. Figure 3). In all NTPDases, the sequences ACR1 and ACR 4 are situated between two $\beta$ strands. The alignment not only implies considerable structural conservation between E-NTPDases and members of the actin/HSP70/sugar kinase superfamily. It also suggests that NTPDases - in spite of their membrane anchorage - may consist of two major domains that repeat basic topology and key conserved sequence domains.

\section{Catalytic properties of heterologously expressed rat NTPDase3}

Preliminary experiments revealed that the ATPase activity at the surface of viable [20] mock-transfected $\mathrm{CHO}$ cells was $8.9 \pm 0.4 \%(n=2$, triplicate determinations in each $)$ of the activity obtained after transfection with the NTPDase3encoding plasmid (500 $\mu \mathrm{M}$ ATP). In the isolated mem-
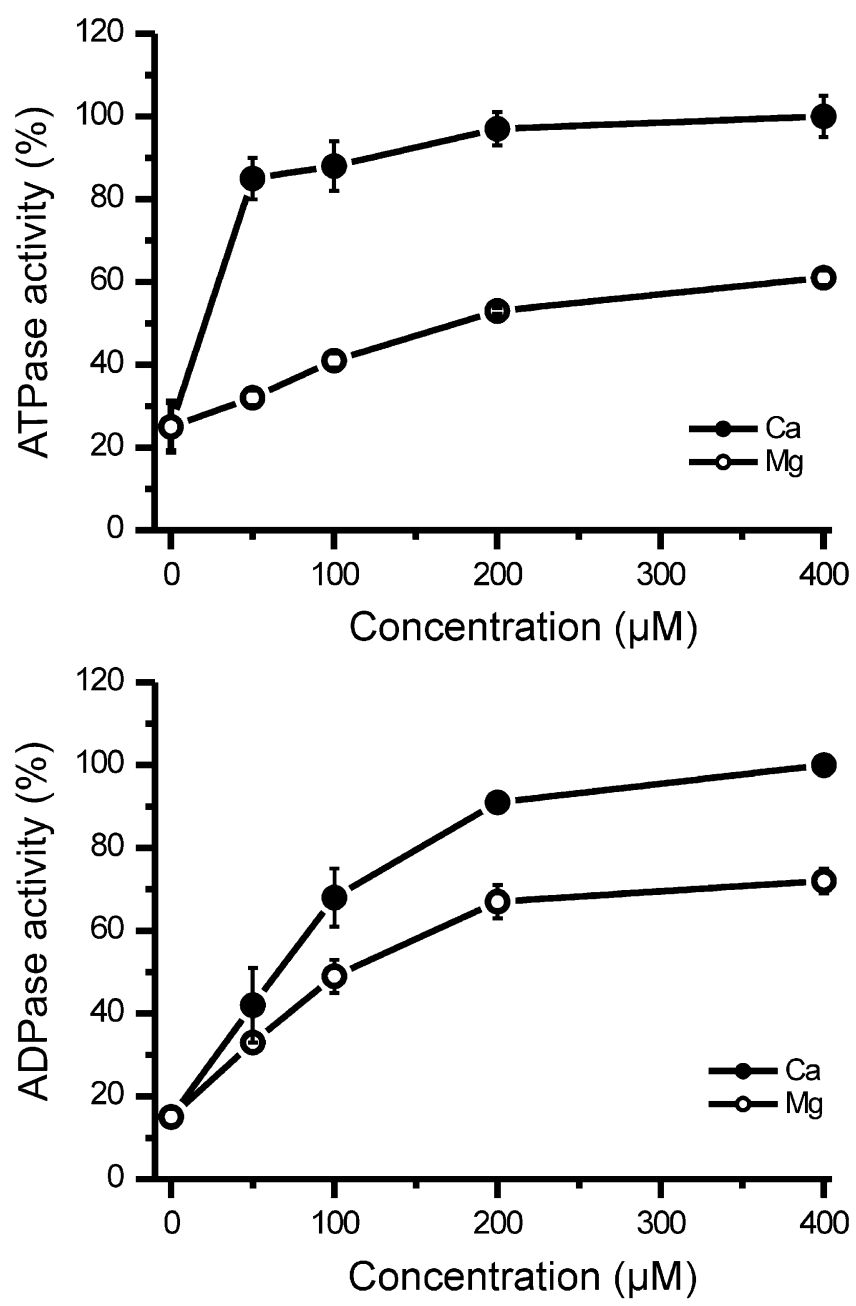

Figure 6. Ion dependence of NTPDase 3 catalytic rate. ATPase (top) or ADPase (bottom) activity of membrane fractions derived from transfected $\mathrm{CHO}$ cells was analyzed in the presence of increasing concentrations of $\mathrm{Ca}^{2+}$ (closed symbol) or $\mathrm{Mg}^{2+}$ (open symbol) $(500 \mu \mathrm{M}$ ATP or ADP). The $100 \%$ values ( \pm S.D., $n=3$ ) correspond to $452 \pm 23$ and $89 \pm 2 \mathrm{nmol} / \mathrm{min} /$ $\mathrm{mg}$ protein for ATPase and ADPase activity, respectively. 
brane fraction, ATPase activity after mock-transfection was $3.1 \pm 2.2 \%(n=7)$ of the activity obtained after NTPDase 3 transfection. No significant ADP hydrolysis could be determined in membrane fractions from mocktransfected cells. In the following, catalytic properties of rat NTPDase 3 were analyzed in membrane fractions, whereby the catalytic activity derived from cells transfected with the empty plasmid was subtracted from that obtained with cDNA-transfected cells. Activity of rat NTPDase 3 strongly depended on the presence of $\mathrm{Ca}^{2+}$ or $\mathrm{Mg}^{2+}$. ATP hydrolysis was stimulated by $\mathrm{Ca}^{2+}$ to a larger extent than by $\mathrm{Mg}^{2+}$, particularly at low concentrations (Figure 6). The difference is less prominent for ADP hydrolysis. In the presence of EDTA $(1 \mathrm{mM})$ no significant nucleotidase activity could be measured (not shown).

ATPase activity revealed an activity optimum between pH 7.5 and 8.5 for both $\mathrm{Ca}^{2+}$ and $\mathrm{Mg}^{2+}$ activation (Figure 7). At $\mathrm{pH} 5$ the rates were approximately $45 \%$ of maximal activity. The following experiments were performed at $\mathrm{pH}$ 7.4 in the presence of $500 \mu \mathrm{M} \mathrm{Mg}^{2+}$ and $500 \mu \mathrm{M}$ nucleotide substrate. An HPLC analysis of product formation using ATP as a substrate showed that ADP is formed in a time-dependent manner and accumulates in the medium. It is further hydrolyzed to AMP. No adenosine is formed (Figure 8).

In addition we compared the hydrolysis rates for ATP, GTP, CTP, ITP and UTP as well as the respective diphosphates. NTPDase3 hydrolyzed nucleoside triphosphates-uridine or pyrimidine nucleotides - at similar rates (Table 1). Nucleoside diphosphates were hydrolyzed at considerably lower rates. In these conditions, the ratio of hydrolysis rates (triphospho- to diphosphonucleotides) varied between 4.1 and 9.5. ATP was hydrolyzed five times faster than ADP and UTP eight times faster than UDP.

\section{Antibody production and enzyme distribution}

A rabbit polyclonal antibody was produced by genetic immunization. The specificity of the antibody was first investigated in $\mathrm{CHO}$ cells transfected with the cDNA encoding NTPDase1, NTPDase2, or NTPDase3 (Figure 9).

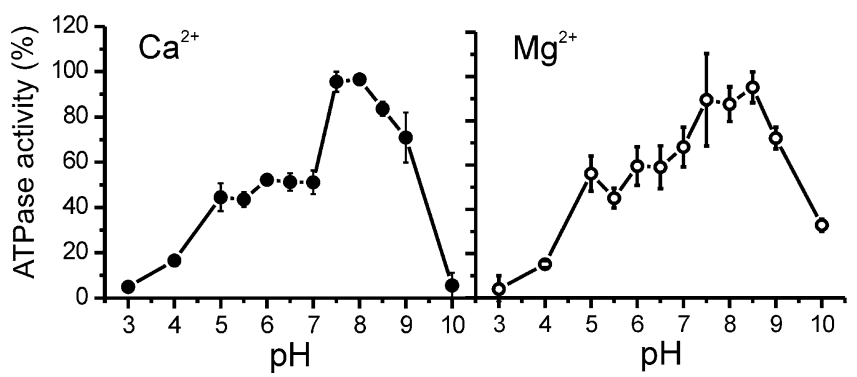

Figure 7. $\mathrm{pH}$ dependence of NTPDase3 catalytic rate. ATPase activity (500 $\mu \mathrm{M}$ ATP) of membrane fractions derived from transfected $\mathrm{CHO}$ cells was analyzed at varying $\mathrm{pH}$ in the presence of either $\mathrm{Ca}^{2+}$ (closed symbol) or $\mathrm{Mg}^{2+}$ (open symbol) $(500 \mu \mathrm{M}$ each). The $100 \%$ values $( \pm$ S.D., $n=3$ ) correspond to $641 \pm 602$ and $370 \pm 339 \mathrm{nmol} / \mathrm{min} / \mathrm{mg}$ protein for $\mathrm{Ca}^{2+}$ and $\mathrm{Mg}^{2+}$-ATPase activity, respectively.

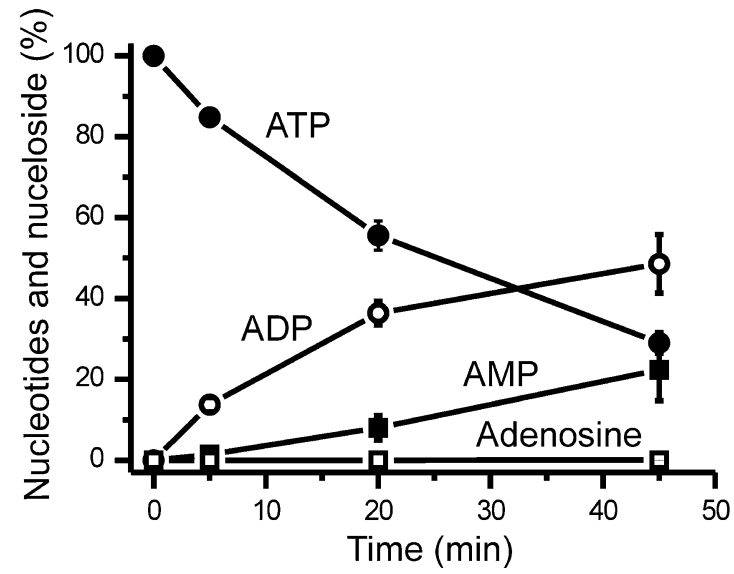

Figure 8. ATP hydrolysis and product formation. ATPase activity (250 $\mu \mathrm{M}$ ATP) of membrane fractions derived from transfected $\mathrm{CHO}$ cells was analyzed at varying time points in the presence of $\mathrm{Ca}^{2+}(250 \mu \mathrm{M})$. ATP hydrolysis and product formation was monitored by HPLC. The contribution of each nucleotide is expressed as percentage of total nucleotides present in the sample. Values of $100 \%$ correspond to the initial amount of ATP substrate. Values represent means \pm SD of three experiments. Equal amounts of starting ATPase activity (7.9 nmol Pi/min) were employed for each assay.

When applied to the surface of viable cells the antiNTPDase3 antibody revealed a significant surface staining of NTPDase3-transfected cells, demonstrating surface localization of the expressed protein. No immunofluorescence was obtained after application of the anti-NTPDase3 antibody to NTPDase1- and NTPDase2-expressing cells. Control experiments verified that antibodies against NTPDase1 or NTPDase2 yielded a cell surface immunosignal with $\mathrm{CHO}$ cells transfected with the respective cDNA (not shown).

Surface staining revealed endogenous expression of NTPDase 3 by the rat-derived PC12 cells (Figure 9e). Immunofluorescence was observed over the entire cell surface. The formation of small immunofluorescent dots implies a partial clustering of the protein. In addition, immunostaining was enhanced at growth cones. Application of preimmune serum either to $\mathrm{CHO}$ or $\mathrm{PC} 12$ cells yielded negative results (not shown).

NTPDase 3 immunoreactivity could be detected by immunoblotting in all brain regions analyzed. In membrane

Table 1. Substrate specificity of recombinant rat NTPDase3 in membrane fractions of transfected $\mathrm{CHO}$ cells.

\begin{tabular}{lllll}
\hline Substrate & $\begin{array}{l}\text { Activity } \\
\text { (\% of ATPase } \\
\text { activity) }\end{array}$ & Substrate & $\begin{array}{l}\text { Activity } \\
\text { (\% of ATPase } \\
\text { activity) }\end{array}$ & NTP:NDP-ratio \\
\hline ATP & 100.0 & ADP & $20.1 \pm 1.3$ & $5.0: 1$ \\
CTP & $126.2 \pm 21.9$ & CDP & $13.3 \pm 2.4$ & $9.5: 1$ \\
GTP & $103.2 \pm 11.1$ & GDP & $25.0 \pm 3.2$ & $4.1: 1$ \\
ITP & $92.1 \pm 8.8$ & IDP & $20.2 \pm 0.9$ & $4.6: 1$ \\
UTP & $109.5 \pm 20.6$ & UDP & $14.3 \pm 6.0$ & $7.7: 1$ \\
\hline
\end{tabular}

Nucleotidase activity (500 $\mu \mathrm{M}$ ATP) of membrane fractions derived from transfected $\mathrm{CHO}$ cells was analyzed in the presence of $\mathrm{Ca}^{2+}(500 \mu \mathrm{M})$. Values represent means \pm SD of three experiments. For ATP $100 \%$ values correspond to $120 \pm 67 \mathrm{nmol} / \mathrm{min} / \mathrm{mg}$ protein. 
fractions obtained from tissue extracts NTPDase3 was hardly detectable (not shown). However, when the protein was enriched by binding to concanavalin A, clear bands could be visualized at $80 \mathrm{kDa}$ (Figure 10). These protein
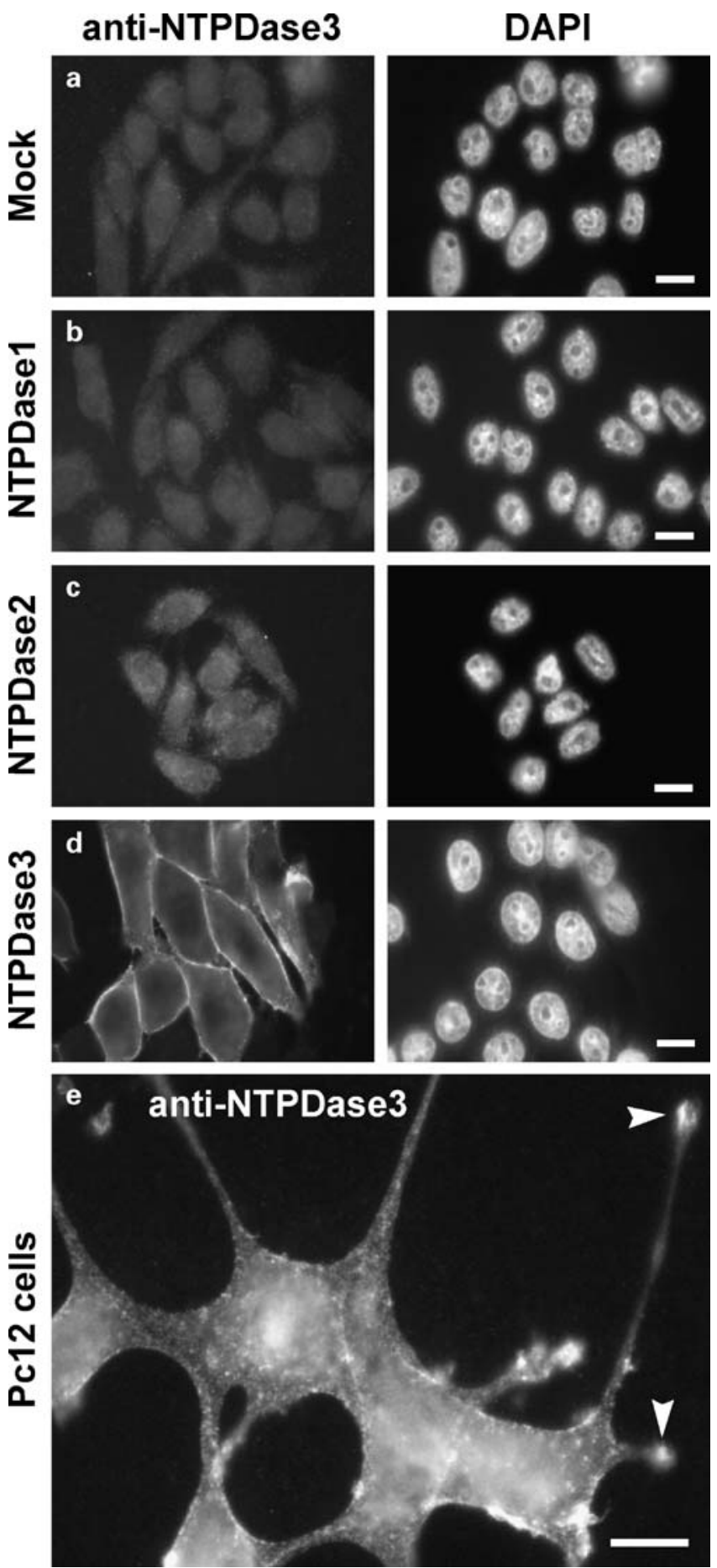

Figure 9. Immunolocalization of NTPDase3. a-d: Viable $\mathrm{CHO}$ cells were incubated with the anti-NTPDase3 antibody two days after transfection with the cDNA encoding the NTPDase indicated at the left or with the empty vector (mock). Antibody binding was visualized by immunofluorescence (left column). Total cells are depicted by DAPI staining of the nuclei (right column). The anti-NTPDase 3 antibody bound only to NTPDase3-transfected cells (d). e: PC12 cells were cultured for 14 days and surface-located immunoreactivity for NTPDase 3 was analyzed as for CHO cells. Arrow heads depict an accumulation of immunoreactivity at growth cones. Bars $=10 \mu \mathrm{m}$.
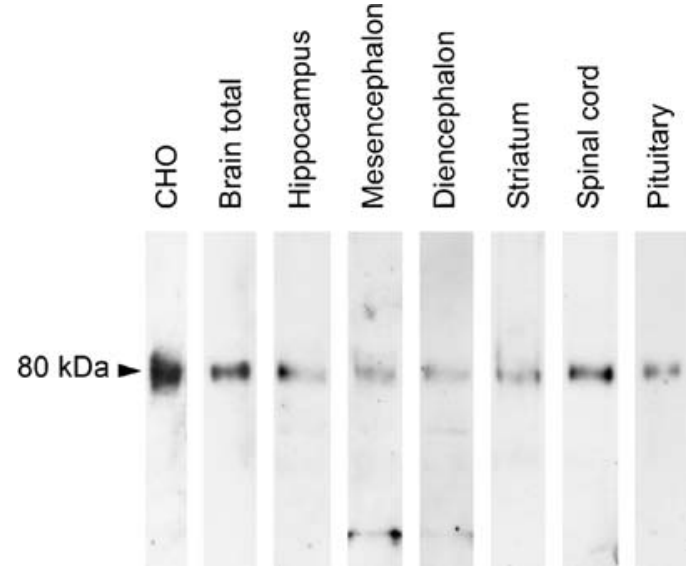

Figure 10. Western blot analysis of NTPDase3 in transfected CHO cells and brain tissue. CHO cells were transfected with NTPDase 3 two days before analysis. The protein amount of ConA-purified membrane fraction loaded per lane corresponded to $25 \mathrm{mg}$ of starting tissue for hippocampus, mesencephalon, diencephalon, striatum and spinal cord, to $10 \mathrm{mg}$ for total brain and $3.3 \mathrm{mg}$ for pituitary.

bands corresponded to that obtained from membrane fractions of CHO cells heterologously expressing NTPDase3. No immunosignal was obtained with $\mathrm{CHO}$ cells transfected with the empty vector or with transfected $\mathrm{CHO}$ cells expressing rat NTPDase1 or rat NTPDase2 (not shown).

\section{Discussion}

Rat NTPDase3 differs significantly in its functional properties from the two other surface-located NTPDases previously isolated from rat, namely NTPDase1 and NTPDase2. Whereas rat NTPDase1 expressed in CHO cells exhibits the typical catalytic properties of an apyrase with a ratio of hydrolysis rates for ATP and ADP of 1:0.8, rat NTPDase2 has dominant ATPase activity with a ratio of 1:0.05-1:0.03 [8, 20, 35]. When expressed in $\mathrm{CHO}$ cells, rat NTPDase3 reveals an ATP to ADP hydrolysis ratio of 1:0.2 and thus represents a functional intermediate between the two other NTPDases. This difference is further underlined when the product formation following hydrolysis of ATP is analyzed. Whereas NTPDase1 hydrolyzes ATP directly to AMP with minimal formation of free ADP, ADP accumulates in the reaction medium following hydrolysis of ATP by NTPDase2 [20]. NTPDase3 reveals intermediate properties. In the presence of ATP, NTPDase 3 accumulates extracellular ADP which is finally converted to AMP. Rat NTPDase3 differs significantly from rat NTPDase1 and NTPDase2 regarding cation dependence. Whereas rat NTPDase1 and NTPDase2 are equally activated by $\mathrm{Ca}^{2+}$ or $\mathrm{Mg}^{2+}$ [20], rat NTPDase3 shows a clear preference for activation by $\mathrm{Ca}^{2+}$. In addition, ATPase and ADPase activities are differentially affected by $\mathrm{Ca}^{2+}$ or $\mathrm{Mg}^{2+}$. This differential activation remains unexplained and may depend on the difference in phosphate chain length between ATP and ADP and thus the potential coordination with the respective metal cation 
within the protein. Removal of divalent cations abolishes catalytic activity of all three enzymes. None of these enzymes hydrolyzes AMP. NTPDase3 shares, however, with NTPDase1 and NTPDase2 its broad substrate specificity towards purine and pyrimidine nucleoside triphosphates. It can be expected that the differences in catalytic properties between individual subtypes of NTPDases differentially affect $\mathrm{P} 2$ receptor signaling either by activating or inactivating P2 receptors [36].

The principal functional properties of rat NTPDase 3 are similar to those of human NTPDase3 (HB6, [22]) and mouse NTPDase3 [23]. Interestingly, the pH-dependence of ATP hydrolysis by rat NTPDase3 clearly differs from that of mouse NTPDase3. Whereas mouse NTPDase3 expresses a considerably higher activity at $\mathrm{pH} 5$ than at pH 7-8 [23], rat NTPDase3 reveals its maximal activity at alkaline $\mathrm{pH}$. Interestingly, the recently cloned NTPDase8 [24] shares principal functional properties with NTPDase3 rather than with NTPDase1 or NTPDase2. Mouse NTPDase 8 is preferentially activated by $\mathrm{Ca}^{2+}$ over $\mathrm{Mg}^{2+}$, has an ATP to ADP hydrolysis ratio of approximately 1:0.5 and accumulates ADP that is effectively further hydrolyzed to AMP [24]. Human NTPDase3 forms a dimer [37] and glycosylation is essential for functional expression [38]. The length of the ORF of rat, mouse [23] and human [22] NTPDase3 is identical (529 aa). The three enzymes share 13 cysteine residues and reveal 7 (rat, human) or 8 (mouse) potential $\mathrm{N}$-glycosylation sites. The rat gene $(31.0 \mathrm{~kb})$ is slightly longer than the mouse gene $(26.9 \mathrm{~kb})$ but it shares its general organization into 11 exons of which exons 2 to 11 contain the ORF.

Rat NTPDase 1 and 2 have previously been immunolocalized in the brain. NTPDase1 is associated with the endothelium of blood vessels and smooth muscle as well as with microglia $[39,40]$. NTPDase 2 is expressed by neural stem cells in the subventricular zone of the lateral ventricles [41]. The immunoblot analysis identified NTPDase 3 in all brain regions investigated. NTPDase3 could be detected by immunocytochemistry at the surface of viable PC12 cells. The corresponding RNA has previously been identified in PC12 cells by RT-PCR together with that of NTPDase 1 and NTPDase2 [42]. Interestingly, the hydrolysis ratio of ATP:ADP (1:0.28) and the product formation following application of ATP to viable PC12 cells is much closer to that of NTPDase3 than of NTPDase2 for which also a weak immunostaining was obtained [42]. This suggests that NTPDase 3 is the predominant ecto-nucleotidase of PC12 cells. In addition we observed an enhancement of NTPDase 3 immunoreactivity at growth cones of $\mathrm{PC} 12$ cells suggesting that the enzyme may be preferentially associated with sites of active membrane incorporation.

The availability of all NTPDase isoforms expected from genomic analysis opens the possibility of structural and functional comparison. To date structural data for this enzyme family are not available. However, the atomic structure of a considerable number of enzymes belonging to the actin/HSP70/sugar kinase superfamily, including glycerol kinase has been derived. All these proteins are soluble, have ATP phosphotransferase or hydrolase activity, depend on divalent metal ion and tend to form oligomeric structures [32]. Individual enzyme families lack global sequence identity. They share, however, the principal structure of two major domains (I and II) of similar folds on either side of a large cleft with an ATP binding site at the bottom of the cleft [30]. These two domains are expected to undergo conformational changes involving movement relative to each other. Both, domain I and II are divided into subdomains (Ia, Ib, IIa, IIb). Subdomains Ia and IIa have the same basic fold with conserved secondary structure elements that share considerable similarity with those of NTPDases.

A comparison of the conserved secondary structure (Figure 5) reveals duplicate conservation of DXG motifs between $\beta$ strands (ACR1 and ACR4) of NTPDases that correspond to the $\beta$ - and $\gamma$-phosphate binding motifs in subdomains Ia and IIa of actin, as well as a conserved glycine in ACR5 that can be identified among all members of the actin/HSP70/sugar kinase superfamily ( $\alpha 3,[32])$. This further supports the notion that E-NTPDases are members of the actin/HSP70/sugar kinase superfamily. It implies in addition that E-NTPDases, like the other members of this superfamily, consist of two major domains with one phosphate binding motif in each domain and the binding of the nucleotide in a cleft between the two opposing domains [31]. Interestingly, some members of the E-NTPDase superfamily are entirely soluble (e.g., potato apyrase or the nucleoside triphosphatases of the protozoan parasite Toxoplasma gondii, references in [35]), others have one transmembrane domain and can be cleaved to form catalytically active soluble enzymes (NTPDase5, NTPDase6), and yet others are (NTPDase 1, 2, 3, 4, 8) are firmly anchored to the membrane via two transmembrane domains. The transmembrane domains of NTPDase 1 were found to be important for maintaining catalytic activity and substrate specificity [6, 43], presumably by affecting tertiary and/or quarternary structure. The two transmembrane domains of NTPDase 1 interact both within and between monomers and may undergo coordinated motions during the process of nucleotide binding and hydrolysis [44].

NTPDases differ from members of the actin/HSP70/ sugar kinase superfamily by additional conserved short sequence domains (ACR2, ACR3). Differences in sequence, secondary and tertiary structure are believed to account for differences in catalytic properties between related NTPDases [35]. The essential role of the ACRs for catalytic activity has been underpinned by a considerable number of studies using point mutations in the ACRs or ACR deletions [2, 29, 45-48].

Our present study shows that rat NTPDase3 displays catalytic properties distinctly different from those of rat NTPDase1 and rat NTPDase2. Rat NTPDase3 differs from mouse NTPDase 3 in its $\mathrm{pH}$ dependence. The enzyme is expressed in multiple brain regions and at the surface of PC12 cells. A comparison of the conserved secondary structure of actin and of NTPDases reveals apparent similarities, inferring that also basic tertiary structure may 
be conserved between members of the actin/HSP70/sugar kinase superfamily and NTPDases.

\section{Acknowledgements}

Supported by grants from the Deutsche Forschungsgemeinschaft (SFB 269, A4) (to N.B., H.Z.) and from the Canadian Institutes of Health Research (to J.S.).

\section{References}

1. Zimmermann H. Extracellular metabolism of ATP and other nucleotides. Naunyn-Schmiedeberg's Arch Pharmacol 2000; 362: 299-309.

2. Zimmermann H. Ectonucleotidases: Some recent developments and a note on nomenclature. Drug Dev Res 2001; 52: 44-56.

3. Handa M, Guidotti G. Purification and cloning of a soluble ATPdiphosphohydrolase (apyrase) from potato tubers (Solanum tuberosum). Biochem Biophys Res Commun 1996; 218: 916-23.

4. Vasconcelos EG, Ferreira ST, de Carvalho TMU et al. Partial purification and immunohistochemical localization of ATP diphosphohydrolase from Schistosoma mansoni - Immunological crossreactivities with potato apyrase and Toxoplasma gondii nucleoside triphosphate hydrolase. J Biol Chem 1996; 271: 22139-45.

5. Smith TM, Kirley TL, Hennessey TM. A soluble ecto-ATPase from Tetrahymena thermophila: Purification and similarity to the membrane-bound ecto-ATPase of smooth muscle. Arch Biochem Biophys 1997; 337: 351-9.

6. Schulte am Esch J, Sévigny J, Kaczmarek E et al. Structural elements and limited proteolysis of CD39 influence ATP diphosphohydrolase activity. Biochemistry 1999; 38: 2248-58.

7. Flaherty KM, Mckay DB, Kabsch W, Holmes KC. Similarity of the three dimensional structures of actin and the ATPase fragment of a 70-kDa heat shock cognate protein. Proc Natl Acad Sci USA 1991; 88: 5041-5.

8. Kegel B, Braun N, Heine P, et al. An ecto-ATPase and an ecto-ATP diphosphohydrolase are expressed in rat brain. Neuropharmacology 1997; 36: 1189-200.

9. Smith TM, Kirley TL. Site-directed mutagenesis of a human brain ecto-apyrase: Evidence that the E-type ATPases are related to the actin/heat shock 70/sugar kinase superfamily. Biochemistry 1999; 38: 321-8.

10. Mulero JJ, Yeung G, Nelken ST, Ford JE. CD39-L4 is a secreted human apyrase, specific for the hydrolysis of nucleoside diphosphates. J Biol Chem 1999; 29: 20064-7.

11. Braun N, Fengler S, Ebeling $\mathrm{C}$ et al. Sequencing, functional expression and characterization of NTPDase6, a nucleoside diphosphatase and novel member of the ecto-nucleoside triphosphate diphosphohydrolase family. Biochem J 2000; 351: 639-47.

12. Hicks-Berger CA, Chadwick BP, Frischauf AM, Kirley TL. Expression and characterization of soluble and membrane-bound human nucleoside triphosphate diphosphohydrolase 6 (CD39L2). J Biol Chem 2000; 275: 34041-5.

13. Wang TF, Guidotti G. Golgi localization and functional expression of human uridine diphosphatase. J Biol Chem 1998; 273: 11392-9.

14. Biederbick A, Rose S, Elsässer HP. A human intracellular apyraselike protein, LALP70, localizes to lysosomal/autophagic vacuoles. J Cell Sci 1999; 112: 2473-84.

15. Biederbick A, Kosan C, Kunz J, Elsässer HP. First apyrase splice variants have different enzymatic properties. J Biol Chem 2000; 275 : 19018-24.

16. Shi JD, Kukar T, Wang CY et al. Molecular cloning and characterization of a novel mammalian endo-apyrase (LALP1). J Biol Chem 2001; 276: 17474-8.
17. Maliszewski CR, DeLepesse GJT, Schoenborn MA et al. The CD39 lymphoid cell activation antigen: Molecular cloning and structural characterization. J Immunol 1994; 153: 3574-83.

18. Kaczmarek E, Koziak K, Sévigny J et al. Identification and characterization of CD39 vascular ATP diphosphohydrolase. J Biol Chem 1996; 271: 33116-22.

19. Wang TF, Guidotti G. CD39 is an ecto- $\left(\mathrm{Ca}^{2+}, \mathrm{Mg}^{2+}\right)$-apyrase. J Biol Chem 1996; 271: 9898-901.

20. Heine P, Braun N, Zimmermann H. Functional characterization of rat ecto-ATPase and ecto-ATP diphosphohydrolase after heterologous expression in CHO cells. Eur J Biochem 1999; 262: 102-7.

21. Mateo J, Harden TK, Boyer JL. Functional expression of a cDNA encoding a human ecto-ATPase. Br J Pharmacol 1999; 128: 396-402.

22. Smith TM, Kirley TL. Cloning, sequencing, and expression of a human brain ecto-apyrase related to both the ecto-ATPases and CD39 ecto-apyrases. Biochim Biophys Acta 1998; 1386: 65-78.

23. Lavoie ÉG, Kukulski F, Lévesque SA et al. Cloning and characterization of mouse nucleoside triphosphate diphosphohydrolase-3. Biochem Pharmacol 2004; 67: 1917-26.

24. Bigonnesse F, Lévesque SA, Kukulski F et al. Cloning and characterization of mouse nucleoside triphosphate diphosphohydrolase-8. Biochemistry 2004; 43: 5511-9.

25. Kukulski F, Lévesque SA, Lavoie ÉG, Lecka J et al. Comparative hydrolysis of P2 receptor agonists by NTPDase 1, 2, 3 and 8. Purinergic Signalling 2005; 1: 193-204.

26. Lanzetta PA, Alvarez LJ, Reinach PS, Candia OA. An improved assay for nanomole amounts of inorganic phosphate. Anal Biochem 1979; 100: 95-7.

27. Liu MA. Overview of DNA vaccines. Ann NY Acad Sci 1995; 772: 15-20.

28. Heilbronn A, Krapohl A, Zimmermann H. 5'-Nucleotidase in PC12 cells as revealed by immunocytochemistry. Cell Tissue Res 1995; 280: 123-31.

29. Murphy DM, Kirley TL. Asparagine 81, an invariant glycosylation site near apyrase conserved region 1 , is essential for full enzymatic activity of ecto-nucleoside triphosphate diphosphohydrolase 3. Arch Biochem Biophys 2003; 413: 107-15.

30. Bork P, Sander C, Valencia A. An ATPase domain common to prokaryotic cell cycle proteins, sugar kinases, actin, and hsp70 heat shock protein. Proc Natl Acad Sci USA 1992; 89: 7290-4.

31. Kabsch W, Holmes KC. The actin fold. FASEB J 1995; 9: 167-74.

32. Hurley JH. The sugar kinase/heat shock protein 70/actin superfamily: Implications of conserved structure for mechanism. Ann Rev Biophys Biomol Struct 1996; 25: 137-62.

33. Kabsch W and Mannherz H-G, Suck D et al. Atomic structure of the actin: DNAaseI complex. Nature 1990; 347: 37-44.

34. Graceffa P, Dominguez R. Crystal structure of monomeric actin in the ATP state. Structural basis of nucleotide-dependent actin dynamics. J Biol Chem 2003; 278: 34172-80.

35. Heine P, Braun N, Sévigny $J$ et al. The C-terminal cysteine-rich region dictates specific catalytic properties in chimeras of the ectonucleotidases NTPDase1 and NTPDase2. Eur J Biochem 2001; 262: 102-7.

36. Ralevic V, Burnstock G. Receptors for purines and pyrimidines. Pharmacol Rev 1998; 50: 413-92.

37. Murphy DM, Ivanenkov VV, Kirley TL. Identification of cysteine residues responsible for oxidative cross-linking and chemical inhibition of human nucleoside-triphosphate diphosphohydrolase 3 . J Biol Chem 2002; 277: 6162-9.

38. Smith TM, Kirley TL. Glycosylation is essential for functional expression of a human brain ecto-apyrase. Biochemistry 1999; 38: 1509-16.

39. Braun N, Sévigny J, Robson SC et al. Assignment of ecto-nucleoside triphosphate diphosphohydrolase-1/cd39 expression to microglia and vasculature of the brain. Eur J Neurosci 2000; 12: 4357-66.

40. Zimmermann H, Braun N, Heine $P$ et al. The molecular and functional properties of E-NTPDase1, E-NTPDase2 and ecto- $5^{\prime}$ nucleotidase in nervous tissue. In van Duffel L, Lemmens R (eds): Ecto-ATPases and Related Ectonucleotidases. Maastricht: Saker Publishing 2000; 9-20.

41. Braun N, Sévigny J, Mishra S et al. Expression of the ecto-ATPase 
NTPDase2 in the germinal zones of the developing and adult rat brain. Eur J Neurosci 2003; 17: 1355-64.

42. Vollmayer P, Koch M, Braun N et al. Multiple ecto-nucleotidases in PC12 cells: Identification and cellular distribution after heterologous expression. J Neurochem 2001; 78: 1019-28.

43. Grinthal A, Guidotti G. Transmembrane domains confer different substrate specificities and adenosine diphosphate hydrolysis mechanisms on CD39, CD39L1, and chimeras. Biochemistry 2002; 41: 1947-56.

44. Grinthal A, Guidotti G. Dynamic motions of CD39 transmembrane domains regulate and are regulated by the enzymatic active site. Biochemistry 2004; 43: 13849-58.

45. Smith TM, Kirley TL. Expression, characterization, and site-directed mutagenesis of a human E-type ATPase. In van Duffel L, Lemmens R (eds): Ecto-ATPases and Related Ectonucleotidases. Maastricht: Shaker Publishing 2000; 90-105.

46. Hicks-Berger CA, Yang F, Smith TM, Kirley TL. The importance of histidine residues in human ecto-nucleoside triphosphate diphosphohydrolase-3 as determined by site-directed mutagenesis. BBA Protein Struct Mol Enzym 2001; 1547: 72-81.

47. Yang F, Hicks-Berger CA, Smith TM, Kirley TL. Site-directed mutagenesis of human nucleoside triphosphate diphosphohydrolase 3 : The importance of residues in the apyrase conserved regions. Biochemistry 2001; 40: 3943-50.

48. Basu S, Murphy-Piedmonte DM, Kirley TL. Conserved lysine residue 79 is important for activity of ecto-nucleoside triphosphate diphosphohydrolase 3 (NTPDase3). Purinergic Signalling 2004; 1: 51-8. 